\title{
Microminerais complexados a aminoácidos no desempenho reprodutivo de matrizes pesadas e resposta da progênie
}

\author{
Complexed trace minerals to amino acids on broiler breeders reproductive performance and \\ response from progeny
}

\author{
Ana Carolina Tozzo Guimarães ${ }^{I}$ Nilva Kazue Sakomura ${ }^{I^{*}}$ Luciano Hauschild $^{\mathrm{I}}$ \\ Bruna Agy Loureiro ${ }^{I}$ Daniella Carolina Zanardo Donato ${ }^{\mathrm{I}}$
}

RESUMO

Este estudo teve como objetivo avaliar o uso de microminerais complexados a aminoácidos na alimentação de matrizes de frango de corte sobre o desempenho, variáveis de incubação e desempenho da progênie. O estudo foi dividido em três experimentos: exp.1. desempenho das matrizes, exp.2. incubação e exp.3. desempenho da progênie. No exp. 1, foram utilizadas 260 aves da linhagem CobbAvian 48 (240 matrizes e 20 machos), avaliando-se produção e massa de ovos, conversão alimentar por massa e por dúzia de ovos, peso e gravidade especifica. No exp. 2, determinou-se a taxa de eclosão, eclodibilidade e fertilidade. No exp. 3 avaliou-se $o$ desempenho dos pintinhos nascidos das incubações. No exp. 1, não houve interação entre tratamento e periodo $(P>0,05)$ para produção de ovos, massa de ovos, conversão alimentar por massa e por dúzia de ovos, peso e gravidade especifica dos ovos. Contudo, houve efeito $(P<0,05)$ do periodo para todas as variáveis citadas anteriormente. No exp. 2, a taxa de eclosão, fertilidade e eclodibilidade não apresentaram diferenças significativas $(P<0,05)$. Com relação ao exp. 3 também não houve diferença significativa $(P<0,05)$ no desempenho $d a$ progênie. A suplementação com microminerais complexados a aminoácidos não influencia no desempenho das matrizes, variáveis de incubação e desempenho das progênies.

Palavras-chave: desempenho, matriz pesada, microminerais complexados, ovo.

\section{ABSTRACT}

This study aimed to evaluate the use of complexed trace minerals to amino acid in broiler breeder feeding evaluating performance, incubation variables and performance of progeny. The study was divided in three trials: exp. 1. broiler breeders performance, exp. 2. incubation and exp. 3. chickens performance. In the exp. 1 two hundred sixty broilers breeders of lineage Cobb Avian 48 (240 females and 20 males) were used. The variables evaluated were egg production and egg mass, feed conversion for egg mass and per dozen eggs, egg weight and specific gravity. In the exp. 2 it was determined rate of hatching, hatchability and fertility. In the exp. 3, it was evaluated the performance of chicks born from incubations. In the exp. 1, there was no interaction between treatment and period $(P>0.05)$ for egg production, egg mass, feed conversion for egg mass and per dozen eggs, egg weight and specific gravity of eggs. However, there was effect $(P<0.05)$ of the period for all variables mentioned above. In the exp. 2, hatching rate, fertility and hatchability did not show significant difference $(P<0.05)$. Regarding the performance of broilers, in the exp. 3 there was no significant difference $(P<0.05)$. Supplementation with complexed trace minerals to amino acids does not influence the performance of breeders, incubation variables and progeny performance.

Key words: broiler breeder, egg, mineral tracer, performance.

\section{INTRODUÇÃO}

A produção brasileira de rações alcançou aproximadamente 64,5 milhões de toneladas e, nesse montante, a avicultura contribuiu com $50 \%$, tornando-se o segmento com maior produção de ração (SINDIRAÇÕES, 2012). Nos programas nutricionais de aves, as rações são balanceadas através de um ajuste entre a oferta de nutrientes dos ingredientes com as exigências nutricionais das aves. Entretanto, nas dietas para matrizes de corte, os microminerais oriundos dos ingredientes

IDepartamento de Zootecnia, Faculdade de Ciências Agrárias e Veterinárias (FCAV), Universidade Estadual Paulista (UNESP), 14884900, Jaboticabal, SP, Brasil. E-mail: sakomura@fcav.unesp.br. *Autor para correspondência. 
não permitem formular uma dieta que atenda às exigências para essa categoria. Um aporte inadequado de microminerais pode comprometer o desempenho das aves, qualidade da casca dos ovos e desempenho da progênie (SECHINATO et al., 2006). Dessa forma, a suplementação de microminerais na dieta tem sido considerada uma prática habitual e obrigatória quando o objetivo é otimizar o desempenho das aves.

As fontes inorgânicas têm sido as mais utilizadas, contudo apresentam menor absorção e biodisponibilidade, quando comparadas às fontes de microminerais complexados a aminoácidos (DOURMAD et al., 2002). GUO et al. (2001) encontraram maior biodisponibilidade estimada para fontes de $\mathrm{Cu}$ quelatado ou complexado a aminoácidos, quando comparadas ao Sulfato de $\mathrm{Cu}$ em experimentos com frangos de corte. Essa melhor biodisponibilidade de microminerais quando quelatados ou complexados a aminoácidos pode permitir o uso de níveis elevados de microminerais na dieta.

Alguns microminerais, como o $\mathrm{Se}$ e Zn, em níveis mais elevados, apresentam efeitos benéficos sobre aspectos reprodutivos em matrizes pesadas e galos, quando em condições de desafios sanitários e de ambiente (SURAI, 2000). Nesse sentido, nutricionistas e produtores, com o objetivo de garantir que as matrizes expressem seu potencial em condições de produção, optam, algumas vezes, por fornecer níveis mais elevados.

Os estudos relacionados a avaliar o efeito de utilizar níveis de microminerais complexados a aminoácidos mais elevados em dietas de matrizes e machos reprodutores são poucos e inconsistentes. No caso de variáveis de incubação, existem poucas informações e, além disso, poucos estudos avaliaram o efeito a longo prazo (matriz, incubação e progênie). Os objetivos deste estudo foram, portanto, avaliar o uso de microminerais complexados a aminoácidos $(\mathrm{Mg}, \mathrm{Cu}, \mathrm{Se}, \mathrm{I}, \mathrm{Fe}$ e $\mathrm{Zn})$ em dietas de fêmeas de matrizes pesadas e machos reprodutores no desempenho das matrizes, parâmetros de incubação e resposta da progênie.

\section{MATERIAL E MÉTODOS}

O presente estudo foi realizado no Setor de Avicultura do Departamento de Zootecnia da Faculdade de Ciências Agrárias e Veterinárias - UNESP, Campus de Jaboticabal - SP. Foram realizados três experimentos: desempenho da matriz, incubação e desempenho da progênie.

\section{Desempenho das matrizes}

O experimento foi conduzido com matrizes em fase de produção da $42^{\text {a }}$ à $58^{\mathrm{a}}$ semana de idade, dividido em 4 ciclos de 28 dias cada. Nesse experimento, foram avaliadas uma dieta contendo micromineral inorgânico e outra com micromineraris complexados a aminoácidos. Foram utilizadas 260 aves da linhagem CobbAvian 48 (240 matrizes e 20 machos reprodutores), com 42 semanas de idade, alojadas em galpão de alvenaria, com boxes telados (área de 3,5×2,0m) e equipado com ventiladores e cortinas laterais para o controle da temperatura. $\mathrm{O}$ delineamento foi em blocos [blocos distribuídos de acordo com a classe de peso: leve $(2,22$ a $2,43 \mathrm{~kg})$, média $(2,44$ a $2,65 \mathrm{~kg})$, padrão $(2,66$ a $2,87 \mathrm{~kg})$, pesada $(2,88$ a 3,09$)$ e super pesada $(3,10$ a 3,31$)]$, com dois tratamentos e duas repetições em cada bloco. A unidade experimental era composta de 13 aves (12 fêmeas e um macho). Os boxes foram equipados com comedouro tubular com grade de restrição para machos e um comedouro posicionado em uma altura mais elevada para evitar o consumo das fêmeas. A água foi fornecida à vontade, em bebedouros tipo nipple. Todos os boxes possuíam um ninho de madeira com seis aberturas.

O suplemento micromineral foi incluído ao nível de $0,1 \%$ na dieta. O suplemento micromineral inorgânico (SMI) era composto por sulfato de manganês (70mg $\mathrm{Mn} \mathrm{kg}^{-1}$ dieta), sulfato de zinco (70mg $\mathrm{Zn} \mathrm{kg}$-1 dieta), sulfato de ferro (60mg Fe kg-1 dieta), sulfato de cobre (10mg $\mathrm{Cu} \mathrm{kg}{ }^{-1}$ dieta), iodato de cálcio ( $1 \mathrm{mg} \mathrm{I} \mathrm{kg}^{-1}$ dieta) e

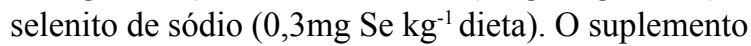
micromineral complexado a aminoácidos (SMCA) foi obtido através da complexação do suplemento micromineral inorgânico em processo industrial. Nesse processo, os íons metálicos provenientes dos sulfatos foram ligados a aminoácidos, oriundos da hidrólise de leveduras, dando origem ao suplemento micromineral complexado a aminoácidos. A diferença entre as dietas dos tratamentos, portanto, consistiu no processo aplicado para complexar os microminerais aos aminoácidos, contudo os níveis de inclusão de microminerais nas dietas foram os mesmos.

Duas dietas, uma para fêmeas (F) e outra para machos $(\mathrm{M})$, foram formuladas para atender as exigências nutricionais da categoria (ROSTAGNO et al., 2005), com base em milho, farelo de soja e farelo de trigo [EM: 2.750 (F) e 2.700 (M) kcal; PB: 15 (F) e 12,6\% (M)]. Os animais foram alimentados com ração farelada todos os dias às $8 \mathrm{~h}$ da manhã. As fêmeas receberam $160 \mathrm{~g}$ de ração dia $^{-1}$ com

Ciência Rural, v.43, n.6, jun, 2013. 
diminuição de $2 \mathrm{~g}$ por semana até atingir 140 gramas. Os machos receberam $135 \mathrm{~g}$ por dia durante todo o período experimental.

Os ovos foram coletados e contabilizados diariamente, assim como o consumo de ração. Nos dois últimos dias de cada ciclo, foi realizada a pesagem dos ovos por parcela para determinação do seu peso médio. A produção de ovos (PD), a massa de ovos (MO), a conversão alimentar por massa de ovos (CAMO) e a conversão alimentar por dúzia de ovos (CADZ) foram determinadas. A qualidade da casca foi mensurada pela gravidade específica (GE), utilizando-se a técnica de imersão dos ovos em soluções salinas com densidades de 1,060 à $1,090 \mathrm{gcm}^{-3}$, com incrementos de 0,005 .

Incubação

Esse experimento foi uma sequência do primeiro com um mesmo delineamento de blocos e com dois tratamentos, contudo com repetição no tempo. Foram realizadas duas incubações, a primeira utilizando os ovos coletados às 46 semanas de idade (338 ovos do SMI e 337 do SMCA) e a segunda, com os ovos coletados às 47 semanas (375 ovos do SMI e 370 do SMC). Cada bloco teve 10 repetições (5 por incubação). Os ovos foram coletados cinco vezes ao dia, durante cinco dias, identificados, pulverizados com a solução AVT $500\left(1,5 \mathrm{mlL}^{-1}\right.$ de água) e armazenados em sala climatizada $\left(19^{\circ} \mathrm{C}\right.$ e $75 \%$ de umidade relativa), por um período de no máximo cinco dias até a incubação. A fertilidade foi avaliada no $21^{\circ}$ dia de incubação, pela contagem dos pintos nascidos, e avaliação dos ovos não eclodidos. Para a verificação dos ovos fertilizados, fez-se a quebra de todos os ovos não eclodidos, calculando-se assim a taxa de eclosão, fertilidade e a eclodibilidade.

\section{Desempenho da progênie}

Os pintos provenientes das duas incubações foram alojados em galpão experimental para frangos de corte em períodos diferentes para avaliar o desempenho através da realização de dois experimentos. Foi utilizado um delineamento inteiramente casualizado, com dois tratamentos (pintos provenientes de matrizes alimentadas com SMI e outros provenientes de matrizes alimentadas com SMCA), com repetição no tempo, 12 repetições (6 em cada período) de 20 aves em cada unidade experimental. Todas as aves receberam as mesmas dietas (pré-inicial, crescimento e terminação), que foram formuladas para atender as exigências nutricionais de cada fase (ROSTAGNO et al., 2005). O desempenho das aves foi avaliado com 7, $21 \mathrm{e}$
42 dias de idade. Foi determinado consumo médio de ração (CMR), ganho de peso (GP) e conversão alimentar (CA).

Análises estatísticas

Os dados do experimento de desempenho das matrizes (PD, MO e CAMO e CADZ) e qualidade de ovos (GE e peso de ovo) foram observados em dois tratamentos e quatro tempos $(28,56,84$ e 112 dias) e analisados por medidas repetidas no tempo através do procedimento ProcMixed do SAS (SAS, 2003). Foi incluído no modelo o bloco como fator de efeito aleatório e tratamento $(\mathrm{T})$, período $(\mathrm{P})$ e a interação $\mathrm{T}^{*} \mathrm{P}$ como fatores de efeitos fixos. A análise foi realizada utilizando a estrutura da matriz de covariâncias entre tempos (S) mais adequada aos dados experimentais. Os dados do experimento de incubação (taxa de eclosão, eclodibilidade e fertilidade dos ovos) e de resposta das progênies (consumo, ganho e conversão) foram submetidos à análise de variância e teste $\mathrm{F}$, para comparação de médias em nível de $5 \%$ de probabilidade. Na análise dos dados de incubação, no modelo, foi considerado o efeito de blocos (peso vivo), tratamentos e incubação. $\mathrm{Na}$ análise dos dados das progênies, no modelo, foi considerado o efeito de tratamento e incubação. As análises foram realizadas utilizando o procedimento PROC GLM do SAS (SAS, 2003).

\section{RESULTADOS E DISCUSSÃO}

O desempenho das aves no presente estudo apresentou valores esperados para produção de ovos e peso das aves, segundo o manual da linhagem. Para as variáveis, produção de ovos, peso dos ovos, massa de ovos, conversão alimentar por massa e por dúzia de ovos e gravidade específica, não foram verificadas diferenças entre os tratamentos $(\mathrm{P}>0,05)$ (Tabela 1 e 2). Na análise de medidas repetidas no tempo, não houve interação entre tratamento e período $(\mathrm{P}>0,05)$ para nenhuma dessas variáveis. Contudo, houve efeito $(\mathrm{P}<0,0001)$ do período para todas as variáveis, exceto para a gravidade específica $(\mathrm{P}=0,40)$. Essas diferenças nos períodos estão relacionadas ao sistema endócrino da ave, que está em constante mudança na produção e secreção de hormônios reprodutivos durante a maturidade sexual (RENEMA et al., 1999). O estudo de HUDSON et al. (2004) também confirma que matrizes apresentam produção de ovos diferenciada no tempo e que o peso dos ovos muda de acordo com a idade das aves. 
Tabela 1 - Produção de ovos (\%/ave/dia), massa de ovos (g/ave/dia) e peso médio dos ovos (g) de matrizes alimentadas com rações com suplemento micromineral inorgânico ou com microminerais complexados a aminoácidos (MCAA).

\begin{tabular}{|c|c|c|c|}
\hline \multirow{2}{*}{ Fatores } & \multirow[b]{2}{*}{ Produção de ovos } & \multirow[b]{2}{*}{ Massa de ovos } & \multirow[b]{2}{*}{ Peso médio dos ovos } \\
\hline & & & \\
\hline Tratamento $(\mathrm{T})$ & $\left(\% \mathrm{ave}^{-1} \mathrm{dia}^{-1}\right)$ & $\left(\right.$ gave $\left.^{-1} \mathrm{dia}^{-1}\right)$ & (g) \\
\hline Inorgânico & 69,13 & 46,43 & 67,20 \\
\hline MCAA & 67,63 & 45,69 & 67,62 \\
\hline \multicolumn{4}{|l|}{ Período (P) } \\
\hline $42-45$ & 76,75 & 51,01 & 66,48 \\
\hline $46-49$ & 72,82 & 49,04 & 67,33 \\
\hline $50-53$ & 65,23 & 44,15 & 67,68 \\
\hline $54-57$ & 58,73 & 40,05 & 68,15 \\
\hline F para tratamento & $0,93(\mathrm{P}=0,34)$ & $\begin{array}{l}\text { Probabilidades--- } \\
0,07(\mathrm{P}=0,79)\end{array}$ & $0,24(\mathrm{P}=0,63)$ \\
\hline F para período & $217,54(\mathrm{P}<0,0001)$ & $148,12(\mathrm{P}<0,0001)$ & $6,36(\mathrm{P}=0,002)$ \\
\hline $\mathrm{F}$ para int. trat. $\mathrm{x}$ tempo & $0,49(\mathrm{P}=0,70)$ & $0,15(\mathrm{P}=0,93)$ & $0,77(\mathrm{P}=0,52)$ \\
\hline Estrutura da matriz (? ) & Compound Symmetry & Huynh-Feldt & Huynh-Feldt \\
\hline
\end{tabular}

O Zn é um componente da enzima anidrase carbônica, que é fundamental para o fornecimento de íons carbonato durante a formação da casca. A inibição dessa enzima resulta na diminuição da secreção do íon bicarbonato e pode reduzir o peso da casca (NYS et al., 2004). Além do $\mathrm{Zn}$, outros microminerais como o $\mathrm{Mn}, \mathrm{Cu}, \mathrm{Fe}$ e Se influenciam no metabolismo da formação do ovo, sendo que a magnitude desse efeito pode ser determinada pelos níveis e forma de suplementação (HUDSON et al., 2004). Contudo, em estudos recentes, não foi observado efeito da suplementação inorgânica de $\mathrm{Zn}, \mathrm{Mn}, \mathrm{Cu}, \mathrm{Fe}$ e
Se ou com esses microminerais complexados a aminoácidos em parâmetros produtivos de poedeiras (SECHINATO et al., 2006).

Maiores efeitos da suplementação de microminerais têm sido observados na qualidade dos ovos. Fontes orgânicas de Zn para matrizes pesadas melhoram a gravidade específica, resistência da casca e porcentagem de casca (HUDSON et al., 2004). Entretanto, SECHINATO et al. (2006), avaliando microminerais complexados a aminoácidos (Zn, Mn, $\mathrm{Cu}, \mathrm{Fe}$ e $\mathrm{Se}$ ), não observaram melhora na gravidade específica para poedeiras. Resultado semelhante

Tabela 2 - Conversão alimentar por massa dos ovos (CAMO), conversão alimentar por dúzia de ovos (CADZ) e gravidade específica dos ovos de matrizes alimentadas com rações com suplemento micromineral inorgânico ou com microminerais complexados a aminoácidos (MCAA).

\begin{tabular}{|c|c|c|c|}
\hline \multirow{2}{*}{ Fatores } & \multirow[b]{2}{*}{ CAMO } & \multirow[b]{2}{*}{ CADZ } & \multirow[b]{2}{*}{ Gravidade específica } \\
\hline & & & \\
\hline \multicolumn{4}{|l|}{ Tratamento $(\mathrm{T})$} \\
\hline Inorgânico & 3,32 & 2,68 & 1,080 \\
\hline MCAA & 3,38 & 2,74 & 1,080 \\
\hline \multicolumn{4}{|l|}{ Período (P) } \\
\hline $42-45$ & 3,24 & 2,58 & 1,080 \\
\hline $46-49$ & 3,21 & 2,59 & 1,080 \\
\hline $50-53$ & 3,39 & 2,75 & 1,080 \\
\hline $54-57$ & 3,56 & 2,90 & 1,080 \\
\hline F para tratamento & $0,40(\mathrm{P}=0,53)$ & $\begin{array}{c}\text { obabilidades--------. } \\
0,11(\mathrm{P}=0,74)\end{array}$ & $1,00(P=0,32)$ \\
\hline F para período & $23,91(\mathrm{P}<0,0001)$ & $42,59(\mathrm{P}<0,0001)$ & $1,00(\mathrm{P}=0,4074)$ \\
\hline $\mathrm{F}$ para int. trat. $\mathrm{x}$ tempo & $0,29(\mathrm{P}=0,83)$ & $0,62(\mathrm{P}=0,61)$ & $1,00(\mathrm{P}=0,4074)$ \\
\hline Estrutura da matriz (? ) & CompoundSymmetry & Huynh-Feldt & Simple \\
\hline
\end{tabular}

Ciência Rural, v.43, n.6, jun, 2013. 
foi encontrado no presente estudo, em que o uso de microminerais complexados a aminoácidos não resultou em melhora na gravidade específica do ovo. Esse efeito pode estar relacionado ao estado nutricional das aves, as quais receberam um nível adequado de suplementação, e ao reduzido desafio sanitário a que as aves foram submetidas. Outro aspecto é que diferenças no processamento de complexação de microminerais a aminoácidos podem gerar efeitos diferenciados, uma vez que a biodisponibilidade dos microminerais está estreitamente relacionada com a forma de processamento.

As taxas de eclosão, fertilidade e eclodibilidade não diferiram $(\mathrm{P}>0,05)$ entre os tratamentos (Tabela 3). Os valores observados de eclosão e eclodibilidade neste experimento foram acima de 88 e 96\%, o que reflete em práticas adequadas de manejo sanitário no matrizeiro e no incubatório. No que se refere à fertilidade, índices elevados são dependentes do potencial do ovo para ser fertilizado (fêmeas) e do potencial de fertilização do esperma (machos). Nesse contexto, alguns microminerais, como $\mathrm{Zn}$, podem ter uma função importante na manutenção reprodutiva das aves. Da mesma forma que os resultados desse estudo, HUDSON et al. (2004) também não observaram efeito da suplementação de microminerais complexados a aminoácidos na taxa de eclosão, fertilidade e eclodibilidade.

O consumo, ganho de peso e conversão alimentar das progênies não diferiram $(\mathrm{P}>0,05)$ entre tratamentos em nenhuma das fases (Tabela 4). Com exceção da conversão alimentar aos 21 e 42 dias de idade, houve efeito da incubação para as demais variáveis $(\mathrm{P}<0,0001)$. Essa diferença devida à idade de incubação dos ovos, no desempenho dos pintos, pode estar relacionada ao peso dos ovos. O aumento do peso dos ovos com a idade das aves influencia no peso dos pintainhos e no seu posterior desempenho. Ao contrário do que acontece com a energia e a proteína, o conteúdo micromineral de um ovo e consequentemente a disponibilidade para os embriões é dependente do seu fornecimento na dieta das matrizes (KIDD et al., 1992). Os minerais complexados, por apresentarem melhor biodisponibilidade quando incluídos em dietas, atendem melhor os sistemas bioquímicos das células do animal, melhoram a resposta imune, gerenciam o estresse oxidativo e desenvolvimento de tecidos e ossos (PAPPAS et al., 2006). Dessa forma, os microminerais como $\mathrm{Zn}$, Mn e Se, quando complexados a aminoácidos, permitem melhora na viabilidade e qualidade dos pintainhos, contudo, dependendo do estado sanitário, não influenciam no desempenho, quando comparado à forma inorgânica (SURAI, 2000).

A maior biodisponibilidade dos microminerais complexados a aminoácidos possibilita a redução do nível de inclusão sem prejuízo na produção e com redução no impacto ambiental, devido à menor excreção desses elementos. Quando em níveis mais elevados, próximos aos normalmente utilizados na forma inorgânica, os microminerais complexados a aminoácidos não deprimem o desempenho das aves, variáveis de incubação e resposta das progênies. Nesse sentido, poderão ser utilizados como estratégia preventiva em situações de desafios sanitários e ambiental.

\section{CONCLUSÃO}

A suplementação de microminerais complexados a aminoácidos em níveis elevados em dietas para matrizes pesadas não altera o desempenho produtivo, variáveis de incubação e desempenho da progênie.

Tabela 3 - Taxa de eclosão, eclodibilidade e fertilidade dos ovos de matrizes e galos alimentados com rações com suplemento micromineral inorgânico ou com microminerais complexados a aminoácidos (MCAA).

\begin{tabular}{llccc}
\hline Estatísticas & Tratamento & Taxa de eclosão (\%) & Eclodibilidade (\%) & Fertilidade (\%) \\
\hline \multirow{2}{*}{ Médias } & Inorgânico & 91,49 & 93,38 & 97,98 \\
& MCAA & 90,37 & 93,67 & 96,45 \\
CV $(\%)^{1}$ & Média & 90,93 & 93,52 & 97,21 \\
F tratamento & & 3,98 & 2,86 & 2,39 \\
\end{tabular}

${ }^{1}$ Coeficiente de variação.

Ciência Rural, v.43, n.6, jun, 2013. 
Tabela 4 - Consumo de ração e ganho de peso total e conversão no período das progênies de matrizes alimentadas com rações com suplemento micromineral inorgânico ou com microminerais complexados a aminoácidos (MCAA) aos 7, 21 e 42 dias de idade, nas duas incubações.

\begin{tabular}{|c|c|c|c|c|c|}
\hline \multirow{3}{*}{ Variáveis } & ------------T & ------------ & \multirow{3}{*}{$\mathrm{CV}^{1}$} & \multirow{3}{*}{\multicolumn{2}{|c|}{ F incubação }} \\
\hline & & & & & \\
\hline & Inorgânico & MCAA & & & \\
\hline Consumo, kg & 0,148 & 0,150 & 6,74 & $0,59(\mathrm{P}=0,44)$ & $23,9(\mathrm{P}<0,0001)$ \\
\hline Ganho de peso, $\mathrm{kg}$ & 0,130 & 0,129 & 3,27 & $1,81(\mathrm{P}=0,185)$ & $8,94(\mathrm{P}=0,004)$ \\
\hline Conversão & 1,13 & 1,16 & 7,59 & $2,48(\mathrm{P}=0,122)$ & $45,51(\mathrm{P}<0,0001)$ \\
\hline Consumo, kg & 0,858 & 0,846 & 10,68 & $1,11(\mathrm{P}=0,297)$ & $193,4(\mathrm{P}<0,0001)$ \\
\hline Ganho de peso, $\mathrm{kg}$ & 0,713 & 0,712 & 9,85 & $0,11(\mathrm{P}=0,737)$ & $377,6(\mathrm{P}<0,0001)$ \\
\hline Conversão & 1,205 & 1,187 & 4,97 & $1,01(\mathrm{P}=0,321)$ & $0,16(\mathrm{P}=0,689)$ \\
\hline Consumo, kg & 4,142 & 4,213 & 4,68 & $3,66(\mathrm{P}=0,062)$ & $60,14(\mathrm{P}<0,0001)$ \\
\hline Ganho de peso, $\mathrm{kg}$ & 2,341 & 2,379 & 4,66 & $2,91(\mathrm{P}=0,095)$ & $45,43(\mathrm{P}<0,0001)$ \\
\hline Conversão & 1,77 & 1,77 & 2,53 & $0,02(\mathrm{P}=0,877)$ & $0,36(\mathrm{P}=0,552)$ \\
\hline
\end{tabular}

${ }^{1}$ Coeficiente de variação

\section{COMITÊ DE ÉTICA E BIOSSEGURANÇA}

Protocolo n. 006138/12.

\section{REFERÊNCIAS}

DOURMAD, J.-Y. et al.Bioavailability of two sources of zinc in weanling pigs. Animal Research, v.51, p.315-326, 2002. Disponível em: <http://dx.doi.org/10.1051/animres:2002028>. Acessoem:25fev. 2012.doi: 10.1051/animres:2002028.

GUO, R. et al.Chemical characteristics and relative bioavailability of supplemental organic copper sources for poultry. Journal of Animal Science, v.79, p.1132-1141, 2001. Disponível em: <http:// jas.fass.org/content/79/5/1132>. Acesso em:25fev. 2012.

HUDSON, B.P. et al. Reproductive performance and immune status of caged broiler breeder hens provided diets supplemented with either inorganic or organic sources of zinc from hatching to 65 wk of age. Journal of Applied Poultry Research, v.13, p.349359, 2004. Disponível em: <http://japr.fass.org/content/13/2/349. short>. Acesso em: 25 fev. 2012.

KIDD, M.T. et al. Progeny performance when dams and chicks are fed supplemental zinc. Poultry Science, v.71, p.1201-1206, 1992. Disponível em: <http://ps.fass.org/content/71/7/1201.abstract>. Acessoem: 25 fev. 2012. doi: 10.3382/ps.0711201.

NYS, Y. et al.Avian eggshell mineralization: biochemical and functional characterization of matrix proteins.Comptes Rendus Palevol, v.3, p.549-562, 2004. Disponível em: <http://www. sciencedirect.com/science/article/pii/S1631068304001393>. Acesso em: 25fev. 2012. doi: 10.1016/j.crpv.2004.08.002.
PAPPAS, A.C. et al. Effects of supplementing broiler breeder diets with organoselenium compounds and polyunsaturated fatty acids on hatchability. Poultry Science, v.85, p.1584-1593, 2006. Disponível em: <http://ps.fass.org/content/85/9/1584.long $>$. Acesso em: 25 fev. 2012.

RENEMA, R. et al. Effects of body weight and feed allocation during sexual maturation in broiler breeder hens. 2. Ovarian morphology and plasma hormone profiles. Poultry Science, v.78, p.629-639, 1999. Disponível em: <http://ps.fass.org/ content/78/5/629.abstract>. Acesso em:25fev. 2012.

ROSTAGNO, H.S. et al.Tabelas brasileiras para aves e suínos. Composição de alimentos e exigências nutricionais. 2 ed. Viçosa, MG: UFV, 2005. 186p.

SAS INSTITUTE.Sasuser'sguide: statistics.Cary, 2003. 846p.

SECHINATO, A.D.S. et al.Efeito da suplementação dietética com micro minerais orgânicos na produção de galinhas poedeiras. Brazilian Journal of Veterinary Research and Animal Science, v.43,p.159-166, 2006.Disponível em:<http://www.revistasusp.sibi. usp.br/scielo.php?pid=S1413-95962006000200003\&script $=$ sci arttext>. Acesso em:25fev. 2012.

SINDIRAÇÕES. Indústria da alimentação animal. Boletin Informativo do Setor,maio, 2012. Disponível em: <http:// sindiracoes.org.br/wp-content/uploads/2011/12/boletim sindiracoes_DEZ2011.pdf>. Acesso em: 21ago. 2012.

SURAI, P.F. Effect of selenium and vitamin E content of the maternal diet on the antioxidant system of the yolk and the developing chick. British Poultry Science, v.41, p.235243, 2000. Disponível em: <http://www.tandfonline.com/doi/ abs/10.1080/713654909\#preview>. Acesso em: 25 fev. 2012. doi: $10.1080 / 713654909$. 